International Journal of Pure and Applied Mathematics

Volume 108 No. 1 2016, 49-62

ISSN: 1311-8080 (printed version); ISSN: 1314-3395 (on-line version)

url: http://www.ijpam.eu

doi: 10.12732/ijpam.v108i1.7

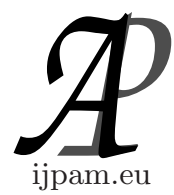

\title{
COUPLED FIXED POINT THEOREMS IN PARTIALLY ORDERED MULTIPLICATIVE METRIC SPACE AND ITS APPLICATIONS
}

\author{
Laishram Shanjit ${ }^{1 \S}$, Yumnam Rohen ${ }^{2}$ \\ Thokchom Chhatrajit Singh ${ }^{3}$, P.P. Murthy ${ }^{4}$ \\ 1,2,3 Department of Basic Sciences and Humanities \\ Natonal Institute of Technology Manipur \\ Langol, 795004, INDIA \\ ${ }^{4}$ Department of Pure and Applied Mathematics \\ Guru Ghasidas Vishwavidyalaya (A Central University) \\ Koni, Bilaspur (Chhattisgarh), 495009, INDIA
}

\begin{abstract}
In this paper, we introduce the concept of coupled fixed point in partially ordered multiplicative metric space by proving some theorems for the existence and uniqueness of coupled fixed point. Also, we discuss the application to the existence and uniqueness of solution for a periodic boundary value problem.
\end{abstract}

AMS Subject Classification: 46B20, 47A12

Key Words: multiplicative metric space, coupled fixed point, partially ordered set

\section{Introduction and Preliminaries}

The concept of multiplicative calculus, in which the role of addition and subtraction are replaced by multiplication and division was not the interest of researchers for a long time even though it was defned by Michael Grossman and Robert Katz in 1967-1970. But Bashirov and Ozyapici [1] draw the attention of researchers specially in the field of analysis by highlighting various

Received: November 5, 2015

Published: May 31, 2016

${ }^{\S}$ Correspondence author (c) 2016 Academic Publications, Ltd.

url: www.acadpubl.eu 
properties like multiplicative derivatives, multiplicative integrals etc. They also highlighted its application to various topics like Newtonian calculus, semigroups of linear operators, multiplicative spaces, multiplicative differential equations, multiplicative calculus of variation etc. Ozavsar and Cevikel [2] introduced the concept of multiplicative contraction mapping. This is one of the most interesting result because with this concept of multiplicative contraction mapping they introduced the famous Banach contraction principle in multiplicative metric spaces.

In 1987 Guo and Lakshmikantham [3] introduced the concept of coupled fixed point. Later, Bhaskar and Lakshmikantham [4] proved a new fixed point theorem for a mixed monotone mapping in a metric space powered with partial ordered by using a weak contractivity type assumption. For more information about multiplicative metric space one can see the research papers in [5-6] and about coupled fixed point, see the research papers in [7-11] and references there in.

The aim of this paper is to introduced the concept of coupled fixed point in the context of partially ordered multiplicative metric space. On the application part we discuss about the applicability to the existence and uniqueness of solution for a periodic boundary value problem.

Definition 1.1 [1] Let $X$ be a non empty set. A mapping $d: X \times X \rightarrow \mathbb{R}$ is said to be a multiplicative metric if it satisfies the following conditions:

(i) $d(x, y) \geq 1$ for all $x, y \in X$ and $d(x, y)=1$ if and only if $x=y$.

(ii) $d(x, y)=d(y, x)$ for all $x, y \in X$

(iii) $d(x, z) \leq d(x, y) \cdot d(y, z)$ for all $x, y, z \in X$ (multiplicative triangle inequality)

Also, $(X, d)$ is called a multiplicative metric space.

Example 1.2 [2] Let $d^{*}:\left(\mathbb{R}^{+}\right)^{n} \times\left(\mathbb{R}^{+}\right)^{n} \rightarrow \mathbb{R}$ be defined as follows

$$
d^{*}(x, y)=\left|\frac{x_{1}}{y_{1}}\right|^{*} \cdot\left|\frac{x_{2}}{y_{2}}\right|^{*} \ldots\left|\frac{x_{n}}{y_{n}}\right|^{*} .
$$

where $x=\left(x_{1}, x_{2}, \ldots, x_{n}\right), y=\left(y_{1}, y_{2}, \ldots, y_{n}\right) \in\left(\mathbb{R}^{+}\right)^{n}$ and $|.|^{*}: \mathbb{R}^{+} \rightarrow \mathbb{R}^{+}$is defined as follows:

$$
|a|^{*}= \begin{cases}a, & \text { if } a \geq 1 \\ \frac{1}{a}, & \text { if } a \leq 1\end{cases}
$$


Then $\left(\left(\mathbb{R}^{+}\right)^{n}, d^{*}\right)$ is a multiplicative metric space.

Example 1.3 [2] Let $a>1$ be a fixed real number. Then $d_{a}: \mathbb{R}^{n} \times \mathbb{R}^{n} \rightarrow \mathbb{R}$ defined by

$$
d_{a}(w, z)=a^{\sum_{i=1}^{n}\left|w_{i}-z_{i}\right|}
$$

where $w=\left(w_{1}, w_{2}, \ldots, w_{n}\right), z=\left(z_{1}, z_{2}, \ldots, z_{n}\right) \in \mathbb{R}^{n}$.

Obviously, $\left(\mathbb{R}^{n}, d_{a}\right)$ is a multiplicative metric space. We also can extend multiplicative metric $\mathbb{C}^{n}$ by the following definition:

$$
d_{a}(w, z)=a^{\sum_{i=1}^{n}\left|w_{i}-z_{i}\right|}
$$

where $w=\left(w_{1}, w_{2}, \ldots, w_{n}\right), z=\left(z_{1}, z_{2}, \ldots, z_{n}\right) \in \mathbb{C}^{n}$.

Example 1.4 [2] Let $X=\left\{(x, 1) \in \mathbb{R}^{2}: 1 \leq x \leq 2\right\} \bigcup\left\{(1, x) \in \mathbb{R}^{2}: 1 \leq\right.$ $x \leq 2\}$. Consider a mapping $d: X \times X \rightarrow \mathbb{R}$ defined by

$$
d((a, b),(c, d))=\left(\left|\frac{a}{c}\right|^{*} \cdot\left|\frac{b}{d}\right|^{*}\right)^{\frac{1}{3}}
$$

Then $(X, d)$ is a multiplicative metric space.

Definition 1.5 [2] Let $(X, d)$ be a multiplicative metric space, $\left\{x_{n}\right\}$ be a sequence in $X$ and $x \in X$. If for every multiplicative open ball

$$
B_{\varepsilon}(x)=\{y \in X \mid d(x, y)<\varepsilon\}, \varepsilon>1
$$

there exists a natural number $N$ such that $n \geq N$, then $x_{n} \in B_{\varepsilon}(x)$. The sequence $\left\{x_{n}\right\}$ is said to be multiplicative convergent to $x$, denoted by $x_{n} \rightarrow$ $x \quad(n \rightarrow \infty)$

Definition $1.6[2]$ Let $(X, d)$ be a multiplicative metric space and $\left\{x_{n}\right\}$ be a sequence in $X$. The sequence is called a multiplicative Cauchy sequence if it holds that for all $\varepsilon>1$, there exists $N \in \mathbb{N}$ such that $d\left(x_{n}, x_{m}\right)<\varepsilon$ for all $m, n>N$.

Definition $1.7[2]$ Let $(X, d)$ be a multiplicative metric space. A mapping $f: X \rightarrow X$ is called a multiplicative contraction if there exists a real constant 
$\lambda \in[0,1)$ such that $d(f(x), f(y)) \leq d(x, y)^{\lambda}$ for all $x, y \in X$

Definition 1.8 [2] We call a multiplicative metric space complete if every multiplicative Cauchy sequence in it is multiplicative convergent to $x \in X$.

Definition 1.9 [4] Let $(X, \preceq)$ be a partially ordered set and $S: X \times$ $X \rightarrow X$. The mapping $S$ is said to has the mixed monotone property if $S$ is monotone non-decreasing in its first argument and is monotone non-increasing in its second argument, that is, for any $x, y \in X$,

$$
\begin{gathered}
x_{1}, x_{2} \in X, x_{1} \preceq x_{2} \Rightarrow S\left(x_{1}, y\right) \preceq S\left(x_{2}, y\right), \\
y_{1}, y_{2} \in X, y_{1} \preceq y_{2} \Rightarrow S\left(x, y_{1}\right) \succeq S\left(x, y_{2}\right)
\end{gathered}
$$

Definition 1.10 [4] An element $(x, y) \in X \times X$ is called a coupled fixed point of the mapping $S: X \times X \rightarrow X$ if

$$
S(x, y)=x, S(y, x)=y \text {. }
$$

\section{Main Results}

We prove the following coupled fixed point theorems.

Theorem 1. Let $(X, \preceq)$ be a partially ordered set and suppose that there is a multiplicative metric $d$ on $X$ such that $(X, d)$ is multiplicative metric space. Let $S: X \times X \rightarrow X$ be a continuous mapping having the mixed monotone property on $X$. Assume that there exists a $\lambda \in[0,1)$ with

$$
d(S(x, y), S(u, v)) \leq[d(x, u) \cdot d(y, v)]^{\frac{\lambda}{2}}
$$

for each $x \succeq u$ and $y \preceq v$.

If there exist $x_{0}, y_{0} \in X$ such that

$$
x_{0} \preceq S\left(x_{0}, y_{0}\right) \text { and } y_{0} \succeq S\left(y_{0}, x_{0}\right)
$$

then there exist $x, y \in X$ such that

$$
x=S(x, y) \text { and } y=S(y, x) .
$$

If for every $(x, y),\left(x^{*}, y^{*}\right) \in X \times X$, there exists $\left(z_{1}, z_{2}\right) \in X \times X$ such that $\left(S\left(z_{1}, z_{2}\right), S\left(z_{2}, z_{1}\right)\right)$ is comparable with $(S(x, y), S(y, x))$ and $\left(S\left(x^{*}, y^{*}\right), S\left(y^{*}, x^{*}\right)\right)$, then $S$ has a unique coupled fixed point. 
Proof: As $x_{0} \preceq S\left(x_{0}, y_{0}\right)=x_{1}$ (say) and $y_{0} \succeq S\left(y_{0}, x_{0}\right)=y_{1}$ (say), let $x_{2}=S\left(x_{1}, y_{1}\right)$ and $y_{2}=S\left(y_{1}, x_{1}\right)$, we denote

$$
\begin{aligned}
& S^{2}\left(x_{0}, y_{0}\right)=S\left(S\left(x_{0}, y_{0}\right), S\left(y_{0}, x_{0}\right)\right)=S\left(x_{1}, y_{1}\right)=x_{2} \\
& S^{2}\left(y_{0}, x_{0}\right)=S\left(S\left(y_{0}, x_{0}\right), S\left(x_{0}, y_{0}\right)\right)=S\left(y_{1}, x_{1}\right)=y_{2} .
\end{aligned}
$$

Now, by the mixed monotone property of $S$, we have

$$
\begin{aligned}
& x_{2}=S^{2}\left(x_{0}, y_{0}\right)=S\left(x_{1}, y_{1}\right) \succeq S\left(x_{0}, y_{0}\right)=x_{1} \\
& y_{2}=S^{2}\left(y_{0}, x_{0}\right)=S\left(y_{1}, x_{1}\right) \preceq S\left(y_{1}, x_{1}\right)=y_{1}
\end{aligned}
$$

Further, for $n=1,2, \ldots$, we denote,

$$
\begin{aligned}
& x_{n+1}=S^{n+1}\left(x_{0}, y_{0}\right)=S\left(S^{n}\left(x_{0}, y_{0}\right), S^{n}\left(y_{0}, x_{0}\right)\right) \\
& y_{n+1}=S^{n+1}\left(y_{0}, x_{0}\right)=S\left(S^{n}\left(y_{0}, x_{0}\right), S^{n}\left(x_{0}, y_{0}\right)\right)
\end{aligned}
$$

It is easy to verify that

$$
\begin{aligned}
& x_{0} \preceq \quad S\left(x_{0}, y_{0}\right)=x_{1} \preceq S^{2}\left(x_{0}, y_{0}\right)=x_{2} \preceq \ldots \preceq S^{n+1}\left(x_{0}, y_{0}\right) \preceq \ldots, \\
& y_{0} \succeq S\left(y_{0}, x_{0}\right)=y_{1} \succeq S^{2}\left(y_{0}, x_{0}\right)=y_{2} \succeq \ldots \succeq S^{n+1}\left(y_{0}, x_{0}\right) \succeq \ldots .
\end{aligned}
$$

Now, for all $n \in \mathbb{N}$, we have to show that

$$
\begin{aligned}
& d\left(S^{n+1}\left(x_{0}, y_{0}\right), S^{n}\left(x_{0}, y_{0}\right)\right) \leq\left[d\left(S\left(x_{0}, y_{0}\right), x_{0}\right) \cdot d\left(S\left(y_{0}, x_{0}\right), y_{0}\right)\right]^{\frac{\lambda^{n}}{2}} \\
& d\left(S^{n+1}\left(y_{0}, x_{0}\right), S^{n}\left(y_{0}, x_{0}\right)\right) \leq\left[d\left(S\left(y_{0}, x_{0}\right), y_{0}\right) \cdot d\left(S\left(x_{0}, y_{0}\right), x_{0}\right)\right]^{\frac{\lambda^{n}}{2}}
\end{aligned}
$$

Now, for $n=1$, using $x_{0} \preceq S\left(x_{0}, y_{0}\right)$ and $y_{0} \succeq S\left(y_{0}, x_{0}\right)$,

$$
\begin{aligned}
d\left(S^{2}\left(x_{0}, y_{0}\right), S\left(x_{0}, y_{0}\right)\right) & =d\left(S\left(S\left(x_{0}, y_{0}\right), S\left(y_{0}, x_{0}\right)\right), S\left(x_{0}, y_{0}\right)\right) \\
& \leq\left[d\left(S\left(x_{0}, y_{0}\right), x_{0}\right) \cdot d\left(S\left(y_{0}, x_{0}\right), y_{0}\right)\right]^{\frac{\lambda}{2}}
\end{aligned}
$$

Similarly,

$$
d\left(S^{2}\left(y_{0}, x_{0}\right), S\left(y_{0}, x_{0}\right)\right) \leq\left[d\left(S\left(y_{0}, x_{0}\right), y_{0}\right) \cdot d\left(S\left(x_{0}, y_{0}\right), x_{0}\right)\right]^{\frac{\lambda}{2}}
$$

which is true for $n=1$.

For $n=2, S^{2}\left(x_{0}, y_{0}\right) \succeq x_{0}$ and $S\left(y_{0}, x_{0}\right) \preceq y_{0}$, we have

$$
\begin{aligned}
d\left(S^{3}\left(x_{0}, y_{0}\right), S^{2}\left(x_{0}, y_{0}\right)\right)= & d\left[S\left(S^{2}\left(x_{0}, y_{0}\right), S^{2}\left(y_{0}, x_{0}\right)\right),\right. \\
& \left(S\left(S\left(x_{0}, y_{0}\right), S\left(y_{0}, x_{0}\right)\right)\right] \\
\leq & {\left[d\left(S^{2}\left(x_{0}, y_{0}\right), S\left(x_{0}, y_{0}\right)\right) .\right.}
\end{aligned}
$$




$$
\begin{aligned}
& \left.d\left(S^{2}\left(y_{0}, x_{0}\right), S\left(y_{0}, x_{0}\right)\right)\right]^{\frac{\lambda}{2}} \\
\leq \quad & {\left[\left\{d\left(S\left(x_{0}, y_{0}\right), x_{0}\right) \cdot d\left(S\left(y_{0}, x_{0}\right), y_{0}\right)\right\}^{\frac{\lambda}{2}} .\right.} \\
& \left.\left\{d\left(S\left(y_{0}, x_{0}\right), y_{0}\right) \cdot d\left(S\left(x_{0}, y_{0}\right), x_{0}\right)\right\}^{\frac{\lambda}{2}}\right]^{\frac{\lambda}{2}} \\
= & {\left[d^{\lambda}\left(S\left(x_{0}, y_{0}\right), x_{0}\right) \cdot d^{\lambda}\left(S\left(y_{0}, x_{0}\right), y_{0}\right)\right]^{\frac{\lambda}{2}} } \\
= & {\left[d\left(S\left(x_{0}, y_{0}\right), x_{0}\right) \cdot d\left(S\left(y_{0}, x_{0}\right), y_{0}\right)\right]^{\frac{\lambda^{2}}{2}} }
\end{aligned}
$$

Similarly,

$$
d\left(S^{3}\left(y_{0}, x_{0}\right), S^{2}\left(y_{0}, x_{0}\right)\right) \leq\left[d\left(S\left(y_{0}, x_{0}\right), y_{0}\right) \cdot d\left(S\left(x_{0}, y_{0}\right), x_{0}\right)\right]^{\frac{\lambda^{2}}{2}}
$$

which is true for $n=2$.

Let us assume that it is true for $n=k$. Now, using $S^{k}\left(x_{0}, y_{0}\right) \succeq S^{k-1}\left(x_{0}, y_{0}\right)$ and $S^{k}\left(y_{0}, x_{0}\right) \preceq S^{k-1}\left(y_{0}, x_{0}\right)$, we have

$$
\begin{aligned}
& d\left(S^{k+1}\left(x_{0}, y_{0}\right), S^{k}\left(x_{0}, y_{0}\right)\right) \leq\left[d\left(S\left(x_{0}, y_{0}\right), x_{0}\right) \cdot d\left(S\left(y_{0}, x_{0}\right), y_{0}\right)\right]^{\frac{\lambda^{k}}{2}} \\
& d\left(S^{k+1}\left(y_{0}, x_{0}\right), S^{k}\left(y_{0}, x_{0}\right)\right) \leq\left[d\left(S\left(y_{0}, x_{0}\right), y_{0}\right) \cdot d\left(S\left(x_{0}, y_{0}\right), x_{0}\right)\right]^{\frac{\lambda^{k}}{2}}
\end{aligned}
$$

Now, for $n=k+1$, using $S^{k+1}\left(x_{0}, y_{0}\right) \succeq S^{k}\left(x_{0}, y_{0}\right)$ and $S^{k+1}\left(y_{0}, x_{0}\right) \preceq$ $S^{k}\left(y_{0}, x_{0}\right)$, we have

$$
\begin{aligned}
d\left(S^{k+2}\left(x_{0}, y_{0}\right), S^{k+1}\left(x_{0}, y_{0}\right)\right)= & d\left[S\left(S^{k+1}\left(x_{0}, y_{0}\right), S^{k+1}\left(y_{0}, x_{0}\right)\right),\right. \\
& d\left(S\left(S^{k}\left(x_{0}, y_{0}\right), S^{k}\left(y_{0}, x_{0}\right)\right)\right] \\
\leq & {\left[d\left(S\left(x_{0}, y_{0}\right), x_{0}\right) \cdot d\left(S\left(y_{0}, x_{0}\right), y_{0}\right)\right]^{\frac{\lambda^{k+1}}{2}} . }
\end{aligned}
$$

Similarly, we can show that

$$
d\left(S^{k+2}\left(y_{0}, x_{0}\right), S^{k+1}\left(y_{0}, x_{0}\right)\right) \leq\left[d\left(S\left(y_{0}, x_{0}\right), y_{0}\right) \cdot d\left(S\left(x_{0}, y_{0}\right), x_{0}\right)\right]^{\frac{\lambda^{k+1}}{2}}
$$

which is true for $n=k+1$. Hence by the principle of mathematical induction, it is true for all $n \in \mathbb{N}$.

This gives $\left\{S^{n}\left(x_{0}, y_{0}\right)\right\}$ and $\left\{S^{n}\left(y_{0}, x_{0}\right)\right\}$ are Cauchy sequences in $X$. Let $m>n$, then

$$
\begin{aligned}
d\left(S^{m}\left(x_{0}, y_{0}\right), S^{n}\left(x_{0}, y_{0}\right)\right) \leq & d\left(S^{m}\left(x_{0}, y_{0}\right), S^{m-1}\left(x_{0}, y_{0}\right)\right) \ldots . \\
& d\left(S^{n+1}\left(x_{0}, y_{0}\right), S^{n}\left(x_{0}, y_{0}\right)\right)
\end{aligned}
$$




$$
\begin{aligned}
\leq & {\left[d\left(S\left(x_{0}, y_{0}\right), x_{0}\right) .\right.} \\
& \left.d\left(S\left(y_{0}, x_{0}\right), y_{0}\right)\right]^{\frac{\lambda^{m-1}+\lambda^{m-2}+\ldots+\lambda^{n}}{2}} \\
= & {\left[d\left(S\left(x_{0}, y_{0}\right), x_{0}\right) \cdot d\left(S\left(y_{0}, x_{0}\right), y_{0}\right)\right]^{\frac{\lambda^{n}-\lambda^{m}}{2(1-\lambda)}} } \\
< & {\left[d\left(S\left(x_{0}, y_{0}\right), x_{0}\right) \cdot d\left(S\left(y_{0}, x_{0}\right), y_{0}\right)\right]^{\frac{\lambda^{n}}{2(1-\lambda)}} }
\end{aligned}
$$

Letting $n \rightarrow \infty$, we obtain

$$
d\left(S^{m}\left(x_{0}, y_{0}\right), S^{n}\left(x_{0}, y_{0}\right)\right) \rightarrow 1
$$

implies $\left\{S^{n}\left(x_{0}, y_{0}\right)\right\}$ is a Cauchy sequence. Similarly, we can show that $\left\{S^{n}\left(y_{0}, x_{0}\right)\right\}$ is a Cauchy sequence.

Since $X$ is a complete multiplicative metric space, there exists $x, y \in X$ such that

$$
\lim _{n \rightarrow \infty} S^{n}\left(x_{0}, y_{0}\right)=x \text { and } \lim _{m \rightarrow \infty} S^{m}\left(y_{0}, x_{0}\right)=y
$$

Next, we have to show

$$
S(x, y)=x \text { and } S(y, x)=y
$$

Let $\varepsilon>1$. Since $S$ is continuous at $(x, y)$, for a given $\varepsilon^{\frac{1}{2}}>1$, there exists a $\delta>1$ such that $d(x, u) . d(y, v)<\delta$ which implies $d(S(x, y) . S(u, v))<\varepsilon^{\frac{1}{2}}$.

Since $\left\{S^{n}\left(x_{0}, y_{0}\right)\right\} \rightarrow x$ and $\left\{S^{n}\left(y_{0}, x_{0}\right)\right\} \rightarrow y$, for $\eta=\min \left(\varepsilon^{\frac{1}{2}}, \delta^{\frac{1}{2}}\right)>1$ there exists $n_{0}, m_{0}$ such that for $n \geq n_{0}, m \geq m_{0}$,

$$
d\left(S^{n}\left(x_{0}, y_{0}\right), x\right)<\eta \text { and } d\left(S^{m}\left(y_{0}, x_{0}\right), y\right)<\eta
$$

Now, for $n \in \mathbb{N}, n \geq \max \left\{n_{0}, m_{0}\right\}$,

$$
\begin{aligned}
d(S(x, y), x) & \leq d\left(S(x, y), S^{n+1}\left(x_{0}, y_{0}\right)\right) \cdot d\left(S^{n+1}\left(x_{0}, y_{0}\right), x\right) \\
& =d\left(S(x, y), S\left(S^{n}\left(x_{0}, y_{0}\right), S^{n}\left(y_{0}, x_{0}\right)\right)\right) \cdot d\left(S^{n+1}\left(x_{0}, y_{0}\right), x\right) \\
& =\varepsilon^{\frac{1}{2}} \cdot \eta \\
& \leq \varepsilon .
\end{aligned}
$$

This gives $S(x, y)=x$. Similarly, we get $S(y, x)=y$.

Finally, we have prove that $S$ has a unique coupled fixed point. If possible, let us assume that $\left(x^{*}, y^{*}\right) \in X \times X$ be second coupled fixed point of $S$, then we need to show that $d\left((x, y),\left(x^{*}, y^{*}\right)\right)=1$, where 


$$
\lim _{n \rightarrow \infty} S^{n}\left(x_{0}, y_{0}\right)=x \text { and } \lim _{n \rightarrow \infty} S^{m}\left(y_{0}, x_{0}\right)=y .
$$

Case I: If $(x, y)$ is comparable to $\left(x^{*}, y^{*}\right)$ with respect to the ordering in $X \times X$, then for every $n=0,1,2, \ldots,\left(S^{n}(x, y), S^{n}(y, x)\right)=(x, y)$ is comparable to $\left(S^{n}\left(x^{*}, y^{*}\right), S^{n}\left(y^{*}, x^{*}\right)\right)=\left(x^{*}, y^{*}\right)$.

Also,

$$
\begin{aligned}
d\left((x, y),\left(x^{*}, y^{*}\right)\right) & =d\left(x, x^{*}\right) \cdot d\left(y, y^{*}\right) \\
& =d\left(S^{n}(x, y), S^{n}\left(x^{*}, y^{*}\right)\right) \cdot d\left(S^{n}(y, x), S^{n}\left(y^{*}, x^{*}\right)\right) \\
& \leq\left[d\left(x, x^{*}\right) \cdot d\left(y, y^{*}\right)\right]^{\lambda^{n}} \\
& =\left[d(x, y), d\left(x^{*}, y^{*}\right)\right]^{\lambda^{n}} \\
\Rightarrow d\left((x, y),\left(x^{*}, y^{*}\right)\right) & =1 \\
\Rightarrow(x, y) & =\left(x^{*}, y^{*}\right)
\end{aligned}
$$

Case II: If $(x, y)$ is not comparable to $\left(x^{*}, y^{*}\right)$, then there exists an upper bound or lower bound $z=\left(z_{1}, z_{2}\right) \in X \times X$. Then, for all $n=0,1,2, \ldots$, $\left(S^{n}\left(z_{1}, z_{2}\right), S^{n}\left(z_{2}, z_{1}\right)\right)$ is comparable to

$$
\left(S^{n}(x, y), S^{n}(y, x)\right)=(x, y) \text { and }\left(S^{n}\left(x^{*}, y^{*}\right), S^{n}\left(y^{*}, x^{*}\right)\right)=\left(x^{*}, y^{*}\right) .
$$

We have,

$$
\begin{aligned}
d\left((x, y),\left(x^{*}, y^{*}\right)\right) \leq & d\left(\left(S^{n}(x, y), S^{n}(y, x)\right),\left(S^{n}\left(x^{*}, y^{*}\right), S^{n}\left(y^{*}, x^{*}\right)\right)\right. \\
\leq & d\left(\left(S^{n}(x, y), S^{n}(y, x)\right), d\left(S^{n}\left(z_{1}, z_{2}\right), S^{n}\left(z_{2}, z_{1}\right)\right)\right) . \\
& d\left(\left(S^{n}\left(z_{1}, z_{2}\right), S^{n}\left(z_{2}, z_{1}\right)\right), d\left(S^{n}\left(x^{*}, y^{*}\right), S^{n}\left(y^{*}, x^{*}\right)\right)\right) \\
\leq & \left\{\left[d\left(x, z_{1}\right) \cdot d\left(y, z_{2}\right)\right] \cdot\left[d\left(z_{1}, x^{*}\right), d\left(z_{2}, y^{*}\right)\right]\right\}^{\lambda^{n}} \\
& \rightarrow 1 \text { as } n \rightarrow \infty .
\end{aligned}
$$

which implies that $(x, y)=\left(x^{*}, y^{*}\right)$. Hence $S$ has a unique coupled fixed point.

Theorem 2. Let $(X, \preceq)$ be a partially ordered set and suppose there is a multiplicative metric $d$ on $X$ such that $(X, d)$ is a complete multiplicative metric space. Assume that $X$ has the following property:

(a) if a non-decreasing sequence $\left\{x_{n}\right\} \rightarrow x$, then $x_{n} \leq x$ for all $n$

(b) if a non-increasing sequence $\left\{y_{n}\right\} \rightarrow y$, then $y \leq y_{n}$ for all $n$. 
Let $S: X \times X \rightarrow X$ be a mapping having the mixed monotone property on $X$. Assume that there exists a $\lambda \in[0,1)$ with

$$
d(S(x, y), S(u, v)) \leq[d(x, u), d(y, v)]^{\frac{\lambda}{2}}
$$

for each $x \succeq u$ and $y \preceq v$.

If there exist $x_{0}, y_{0} \in X$ such that

$$
x_{0} \preceq S\left(x_{0}, y_{0}\right) \text {, and } y_{0} \succeq S\left(y_{0}, x_{0}\right),
$$

then there exists $x, y \in X$ such that

$$
x=S(x, y) \text { and } y=S(y, x) .
$$

Proof: Obviously, $\left\{S^{n}\left(x_{0}, y_{0}\right)\right\}$ and $\left\{S^{n}\left(y_{0}, x_{0}\right)\right\}$ are Cauchy sequences in $X$. Then we need to show that $x=S(x, y)$ and $y=S(y, x)$.

Let $\varepsilon>1$. Since $\left\{S^{n}\left(x_{0}, y_{0}\right)\right\} \rightarrow x$ and $\left\{S^{n}\left(y_{0}, x_{0}\right)\right\} \rightarrow y$, there exists $n_{1}, n_{2} \in \mathbb{N}$ such that, for all $n \geq n_{1}$ and $m \geq n_{2}$, we have

$$
d\left(S^{n}\left(x_{0}, y_{0}\right), x\right)<\varepsilon^{\frac{1}{3}} \text { and } d\left(S^{m}\left(y_{0}, x_{0}\right), y\right)<\varepsilon^{\frac{1}{3}} .
$$

Taking $n \in \mathbb{N}, n \geq \max \left\{n_{1}, n_{2}\right\}$ and using $S^{n}\left(x_{0}, y_{0}\right) \preceq x, S^{n}\left(y_{0}, x_{0}\right) \succeq y$, we get

$$
\begin{aligned}
d(S(x, y), x) & =\leq d\left(S(x, y), S^{n+1}\left(x_{0}, y_{0}\right)\right) \cdot d\left(S^{n+1}\left(x_{0}, y_{0}\right), x\right) \\
& =d\left(S(x, y), S\left(S^{n}\left(x_{0}, y_{0}\right), S^{n}\left(y_{0}, x_{0}\right)\right)\right) \cdot d\left(S^{n+1}\left(x_{0}, y_{0}\right), x\right) \\
& \leq\left[d\left(x, S^{n}\left(x_{0}, y_{0}\right)\right) \cdot d\left(y, S^{n}\left(y_{0}, x_{0}\right)\right)\right]^{\frac{\lambda}{2}} \cdot d\left(S^{n+1}\left(x_{0}, y_{0}\right), x\right) \\
& \leq\left[d\left(x, S^{n}\left(x_{0}, y_{0}\right)\right) \cdot d\left(y, S^{n}\left(y_{0}, x_{0}\right)\right)\right] \cdot d\left(S^{n+1}\left(x_{0}, y_{0}\right), x\right) \\
& <\varepsilon .
\end{aligned}
$$

This implies that $S(x, y)=x$. Similarly, we get $S(y, x)=y$.

Theorem 3. In addition to the hypothesis of theorem 1, suppose that every pair of elements of $X$ has an upper bound or a lower bound in $X$. Then $x=y$.

Proof: Case I: If $x$ is comparable to $y$, then $x=S(x, y)$ is comparable $y=S(y, x)$ and $0 \leq \lambda<1$, we get

$$
d(x, y)=d(S(x, y), S(y, x))
$$




$$
\begin{aligned}
& \leq\left[d((x, y) \cdot d(y, x)]^{\frac{\lambda}{2}}\right. \\
\Rightarrow d(x, y) & =1 \\
\Rightarrow x & =y .
\end{aligned}
$$

Case 2: If $x$ is not comparable to $y$, then there exists an upper bound or a lower bound of $x$ and $y$. That is, there exists a $z \in X$ comparable to $x$ and $y$. Suppose that $x \leq z, y \leq z$ holds. Then, we have

$$
\begin{aligned}
& S(x, y) \preceq S(z, y) \text { and } S(x, y) \succeq S(x, z) \\
& S(y, x) \preceq S(z, x) \text { and } S(y, x) \succeq S(y, z)
\end{aligned}
$$

By the mixed monotone property of $S$, we have

1. $S^{2}(x, y)=S(S(x, y), S(y, x)) \leq S(S(z, y), S(y, z))=S^{2}(z, y)$ implies $S^{2}(x, y) \leq S^{2}(z, y)$

2. $S^{2}(y, x)=S(S(y, x), S(x, y)) \leq S(S(z, x), S(x, z))=S^{2}(z, x)$ implies $S^{2}(y, x) \leq S^{2}(z, x)$

3. $S^{2}(x, y)=S(S(x, y), S(y, x)) \geq S(S(x, z), S(z, x))=S^{2}(x, z)$ implies $S^{2}(x, y) \geq S^{2}(x, z)$

4. $S^{2}(y, x)=S(S(y, x), S(x, y)) \geq S(S(y, z), S(z, y))=S^{2}(y, z)$ implies $S^{2}(y, x) \geq S^{2}(y, z)$.

We have similar relations for $n>2$. Now,

$$
\begin{aligned}
d(x, y)= & d\left(S^{n+1}(x, y), S^{n+1}(y, x)\right) \\
= & d\left(S\left(S^{n}(x, y)\right), S\left(S^{n}(y, x)\right)\right) \\
= & d\left(S\left(S^{n}(x, y)\right), S\left(S^{n}(y, x)\right),\left(S\left(S^{n}(y, x)\right), S\left(S^{n}(x, y)\right)\right)\right. \\
\leq & d\left(S\left(S^{n}(x, y), S^{n}(y, x)\right), S\left(S^{n}(x, z), S^{n}(z, x)\right)\right) \\
& d\left(S\left(S^{n}(x, z), S^{n}(z, x)\right), S\left(S^{n}(y, x), S^{n}(x, y)\right)\right) \\
= & d\left(S\left(S^{n}(x, y), S^{n}(y, x)\right), S\left(S^{n}(x, z), S^{n}(z, x)\right)\right) \\
& d\left(S\left(S^{n}(x, z), S^{n}(z, x)\right), S\left(S^{n}(z, x), S^{n}(x, z)\right)\right) \\
& d\left(S\left(S^{n}(z, x), S^{n}(x, z)\right), S\left(S^{n}(y, x), S^{n}(x, y)\right)\right)
\end{aligned}
$$

Using the contractive condition on $S$, we get

$$
\begin{aligned}
d(x, y) \leq & {\left[d\left(S^{n}(x, y), S^{n}(x, z)\right) \cdot d\left(S^{n}(y, x), S^{n}(z, x)\right)\right.} \\
& d\left(S^{n}(x, z), S^{n}(z, x)\right) \cdot d\left(S^{n}(z, x), S^{n}(x, z)\right)
\end{aligned}
$$




$$
\begin{aligned}
& \left.d\left(S^{n}(z, x), S^{n}(y, x)\right) \cdot d\left(S^{n}(x, z), S^{n}(x, y)\right)\right]^{\frac{\lambda}{2}} \\
= & {\left[d^{2}\left(S^{n}(x, y), S^{n}(x, z)\right) \cdot d^{2}\left(S^{n}(x, z), S^{n}(z, x)\right) .\right.} \\
& \left.d^{2}\left(S^{n}(z, x), S^{n}(y, x)\right)\right]^{\frac{\lambda}{2}} \\
\leq \quad & {\left[d\left(S^{n}(x, y), S^{n}(x, z)\right) \cdot d\left(S^{n}(x, z), S^{n}(z, x)\right) \cdot d\left(S^{n}(z, x), S^{n}(y, x)\right)\right]^{\lambda} } \\
& \rightarrow 1 \text { as } n \rightarrow \infty . \\
\Rightarrow x= & y .
\end{aligned}
$$

Theorem 4. In addition to the hypothesis of Theorem 1, suppose that $x_{0}, y_{0} \in X$ are comparable, then $x=y$.

\section{Application}

Here periodic boundary value problem is used as an application to show the existence and uniqueness of our main results.

Let

$$
\begin{gathered}
u^{\prime}=h(t, u), t \in I=(0, T) \\
u(0)=u(T)
\end{gathered}
$$

be the periodic boundary value problem.

By [4], let us assume that there exists continuous functions $f, g$ such that

$$
h(t, u)=f(t, u)+g(t, u), t \in[0, T]
$$

where $f, g$ satisfy the following conditions:

Also, let $\lambda_{1}, \lambda_{2}, \lambda_{3}, \lambda_{4}>0$ be such that $\forall u, v \in \mathbb{R}, v \leq u$,

$$
\begin{gathered}
0 \leq\left(f(t, u)+\lambda_{1} u\right)-\left(f(t, v)+\lambda_{1} v\right) \leq \mu_{1}(u-v) \\
-\mu_{2}(u-v) \leq\left(g(t, u)-\lambda_{2} u\right)-\left(g(t, v)-\lambda_{2} v\right) \leq 0
\end{gathered}
$$

where $\frac{\max \left\{\mu_{1}, \mu_{2}\right\}}{\lambda_{1}+\lambda_{2}}<1$.

To obtain the unique solution of equation (3.1) and (3.2), let

$$
\begin{aligned}
& u^{\prime}+\lambda_{1} u-\lambda_{2} v=f(t, u)+g(t, v)+\lambda_{1} u-\lambda_{2} v \\
& v^{\prime}+\lambda_{1} v-\lambda_{2} u=f(t, v)+g(t, u)+\lambda_{1} v-\lambda_{2} u
\end{aligned}
$$


along with the periodic boundary conditions,

$$
u(0)=u(T) \text { and } v(0)=v(T)
$$

Now, writing equation (3.5) to (3.7) in an integral equations as follows:

$$
\begin{aligned}
u(t)= & \int_{0}^{T}\left[G_{1}(t, s)\left[f(s, u)+g(s, v)+\lambda_{1} u-\lambda_{2} v\right]+\right. \\
& \left.G_{2}(t, s)\left[f(s, v)+g(s, u)+\lambda_{1} v-\lambda_{2} u\right]\right] d s \\
v(t)= & \int_{0}^{T}\left[G_{1}(t, s)\left[f(s, v)+g(s, u)+\lambda_{1} v-\lambda_{2} u\right]+\right. \\
& \left.G_{2}(t, s)\left[f(s, u)+g(s, v)+\lambda_{1} u-\lambda_{2} v\right]\right] d s
\end{aligned}
$$

where

$$
\begin{aligned}
& G_{1}(t, s)= \begin{cases}\frac{1}{2}\left[\frac{e^{\sigma_{1}(t-s)}}{1-e^{\sigma_{1} T}}+\frac{e^{\sigma_{2}(t-s)}}{1-e^{\sigma_{2} T}}\right] & \text { if } 0 \leq s<t \leq T \\
\frac{1}{2}\left[\frac{e^{\sigma_{1}(t+T-s)}}{1-e^{\sigma_{1} T}}+\frac{e^{\sigma_{2}(t+T-s)}}{1-e^{\sigma_{2} T}}\right] & \text { if } 0 \leq t<s \leq T\end{cases} \\
& G_{2}(t, s)= \begin{cases}\frac{1}{2}\left[\frac{e^{\sigma_{2}(t-s)}}{1-e^{\sigma_{2} T}}-\frac{e^{\sigma_{1}(t-s)}}{1-e^{\sigma_{1} T}}\right] & \text { if } 0 \leq s<t \leq T \\
\frac{1}{2}\left[\frac{e^{\sigma_{2}(t+T-s)}}{1-e^{\sigma_{2} T}}-\frac{e^{\sigma_{1}(t+T-s)}}{1-e^{\sigma_{1} T}}\right] & \text { if } 0 \leq t<s \leq T\end{cases}
\end{aligned}
$$

where $\sigma_{1}=-\left(\lambda_{1}+\lambda_{2}\right)$ and $\sigma_{2}=\left(\lambda_{2}-\lambda_{1}\right)$.

By lemma 3.2 of [4], if

$$
\ln \left(\frac{2 e-1}{e}\right) \leq\left(\lambda_{2}-\lambda_{1}\right) T \text { and }\left(\lambda_{1}+\lambda_{2}\right) T \leq 1,
$$

then $G_{1}(t, s) \geq 0$ for $0 \leq t, s \leq T$ and $G_{2}(t, s) \leq 0$ for $0 \leq t, s \leq T$.

Let $X=C(I, \mathbb{R})$ be the multiplicative metric space of all continuous functions $u: I \rightarrow \mathbb{R}$ with the multiplicative metric $d$ defined on $X$ by

$$
d(u, v)=a^{\sup _{t \in I}|u(t)-v(t)|}, \forall u, v \in X, a>1 .
$$

For $a>1$, let the metric $d_{1}$ on $X^{2}$ defined by

$$
d_{1}\left(\left(u_{1}, v_{1}\right),\left(u_{2}, v_{2}\right)\right)=a^{\sup _{t \in I}\left|u_{1}(t)-u_{2}(t)\right|} \cdot a^{\sup _{t \in I}\left|v_{1}(t)-v_{2}(t)\right|}
$$

Also, we take partial order relation on $X^{2}$ by 


$$
\left(u_{1}, v_{1}\right) \preceq\left(u_{2}, v_{2}\right) \Leftrightarrow u_{1}(t) \preceq u_{2}(t) \text { and } v_{1}(t) \succeq v_{2}(t), t \in I .
$$

Define $P: X \times X \rightarrow X$, for $t \in I$, by

$$
\begin{aligned}
P[u, v](t)= & \int_{0}^{T}\left[G_{1}(t, s)\left[f(s, u)+g(s, v)+\lambda_{1} u-\lambda_{2} v\right]+\right. \\
& \left.G_{2}(t, s)\left[f(s, v)+g(s, u)+\lambda_{1} v-\lambda_{2} u\right]\right] d s .
\end{aligned}
$$

If $(u, v) \in X^{2}$ is a coupled fixed point of $P$, then we get

$$
u(t)=P[u, v](t) \text { and } v(t)=P[v, u](t) \forall t \in I .
$$

This shows that $(u, v)$ is a solution of the periodic boundary value problem and satisfy the periodic boundary conditions (3.7).

\section{Acknowledgments}

Authors are thankful to the editors and refrees.

\section{References}

[1] Agamieza E. Bashirov, Emine Misirli Kurpinar, Ali Ozyapici, Multiplicative calculus and its applications, J. Math. Anal. Appl., 337 (2008), 36-48.

[2] M. Ozavsar, A.C. Cevikel, Fixed point of multiplicative contraction mappings on multiplicative metric spaces, ArXiv: 1205.5131v1 [math.GM] 2012.

[3] D. Guo, V. Lakshmikantham, Coupled fixed points of nonlinear operators with applications, Nonlinear Analysis, 11 (1987), 623-632.

[4] T. Ghana Bhaskar, V. Lakshmikantham, Fixed point theorems in partially ordered metric spaces and applications, Nonlinear Analysis, 65 (2006), 1379-1393.

[5] Xiaoju He, Meimei Song, Danping Chen, Common fixed points for weak commutative mappings on a multiplicative metric space, Fixed Point Theory and Applications, 2014 (2014), 48.

[6] Oratai Yamaod, Wuthiphol Sintunavarat, Some fixed point results for generalized contraction mappings with cyclic $(\alpha, \beta)$-admissible mappings in multiplicative metric space, Journal of Inequalities and Applications, 2014 (2014), 488.

[7] Ravi P. Agarwal, Wuthiphol Sintunavarat, Poom Kumam, Coupled coincidence point and common coupled fixed point theorems lacking the mixed monotone property, Fixed Point Theory and Applications, 201 (2013), 22.

[8] Zaid Mohammed Fadail, Abd Ghafur Bin Ahmad, Coupled coincidence point and common coupled fixed point results in cone b-metric spaces, Fixed Point Theory and Applications (2013), 177. 
[9] Preeti Kaushik, Sanjay Kumar, Poom Kumam, Coupled coincidence point theorems for $\alpha-\psi$-contractive type mappings in partially ordered metric spaces, Fixed Point Theory and Applications (2013), 325.

[10] Fawzia Shaddad, Mohd Salmi Md Noorani, Saud M. Alsulami, Habibulla Akhadkulov, Coupled point results in partialy ordered metric spaces without compatibility, Fixed Point Theory and Applications (2014), 204.

[11] P. Kumam, V Pragadeeswarar, M. Marudai, K. Sitthithakerngkiet, Coupled best proximity points in ordered metric spaces, Fixed Point Theory and Applications (2014), 107. 